Piotr Szuca, Department of Mathematics, Gdańsk University, Wita Stwosza 57, 80-952 Gdańsk, Poland.

\title{
ON SOME PROPERTIES OF SETS BLOCKING ALMOST CONTINUOUS FUNCTIONS
}

\begin{abstract}
We define operators $\mathcal{E}(\cdot)$ and $\mathcal{N}(\cdot)$ for blocking sets for almost continuous functions. Using the language of these operators we give new proofs for some classical theorems and prove some new theorems. Finally, we make some remarks regarding uniform limits of almost continuous functions.
\end{abstract}

\section{Preliminaries}

Let $\mathbb{I}=[0,1]$. We will consider the AC subclass of Darboux real functions defined on the interval $\mathbb{I}$ (a function $f: A \rightarrow \mathbb{R}$ is AC (almost continuous) if whenever $U \subset A \times \mathbb{R}$ is an open set containing the graph of $f$, then $U$ contains the graph of a continuous function $g: A \rightarrow \mathbb{R}$ ). For properties of this and other Darboux-like classes of functions see e. g. the survey [4]. In particular it is known, that if $f: \mathbb{I} \rightarrow \mathbb{R} \in \mathrm{AC}$ then $f \nmid J$ is almost continuous as a function from $J$ for every interval $J \subset \mathbb{I}$. It is also known, that for every finite set $F \subset \mathbb{I}$ and open neighborhood $G$ of $f$ there exists continuous function $g \subset G$ such that $g\lceil F=f\lceil F$.

For every set $A \subset \mathbb{I} \times \mathbb{R}$ and $x \in \mathbb{I}$, by $A_{x}$ we will denote $\{y \in \mathbb{R} \mid\langle x, y\rangle \in A\}$. By $\operatorname{bd}(A)$ we will denote border of $A$. We will say that set $A$ is left-open (rightopen) iff for every $\langle x, y\rangle \in A$ there exists open neighborhood $U$ of $\langle x, y\rangle$ such that $([0, x] \times \mathbb{R}) \cap U \subset A(([x, 1] \times \mathbb{R}) \cap U \subset A)$.

\footnotetext{
Key Words: Darboux functions, almost continuous functions, blocking set, Baire 1, Cesáro-Vietoris function, maximum of functions, uniform limit.

Mathematical Reviews subject classification: 26A15

Received by the editors June 5, 2001

* Some of the results presented in this paper are taken from author's Master Thesis, written under the supervision of Tomasz Natkaniec.
} 
For an $a \in \mathbb{R}$ and a set $A \subset \mathbb{R}$ by $|a-A|$ we will denote distance between $a$ and $A$.

We will use the symbol $\bar{A}$ for denoting the topological closure of set $A$. We will also use the symbol $\overline{\mathcal{F}}$ for denoting family of all limits of uniformly convergent sequences of functions from $\mathcal{F} \subset \mathbb{R}^{\mathbb{I}}$.

A closed set $B \subset \mathbb{I} \times \mathbb{R}$ is blocking if $f \cap B=\emptyset$ for at least one function $f: \mathbb{I} \rightarrow \mathbb{R}$ and $g \cap B \neq \emptyset$ for every continuous function $g: \mathbb{I} \rightarrow \mathbb{R}$. Obviously, $f$ is almost continuous iff $f \cap B \neq \emptyset$ for every blocking set $B$ (see e. g. [8]).

\section{Properties of blocking sets}

There are known examples of sets blocking almost continuous functions which have some pathological properties (see e. g. [12]). To investigate general properties of such sets we define two operators.

Definition 1. Let $B \subset \mathbb{I} \times \mathbb{R}$ be a blocking set. Define:

- $\mathcal{E}(B)=\{\langle a, b\rangle \in \mathbb{I} \times \mathbb{R} \mid(\exists h:[0, a] \rightarrow \mathbb{R})(h(a)=b \& h \cap B=$ $\emptyset \& h$ is continuous) $\}$;

- $\mathcal{N}(B)=(\mathbb{I} \times \mathbb{R}) \backslash(B \cup \mathcal{E}(B))$.

Note that $B, \mathcal{E}(B), \mathcal{N}(B)$ are pairwise disjoint and $B \cup \mathcal{E}(B) \cup \mathcal{N}(B)=\mathbb{I} \times \mathbb{R}$. Thus every blocking set $B$ divides the plane into three parts $B, \mathcal{E}(B)$ and $\mathcal{N}(B)$-we will show below that these parts have some nice properties.

Definition 2. Let B be a blocking set. Define also:

- $\mathrm{A}_{\mathrm{EE}}(B)=\{\langle a, b\rangle \in \mathbb{I} \times \mathbb{R} \mid$ there exists an open set $G$ such that $\langle a, b\rangle \in$ $G \& G \subset \mathcal{E}(B)\}$;

- $\mathrm{A}_{\mathrm{NN}}(B)=\{\langle a, b\rangle \in \mathbb{I} \times \mathbb{R} \mid$ there exists an open set $G$ such that $\langle a, b\rangle \in$ $G \& G \subset \mathcal{N}(B)\}$

- $\mathrm{A}_{\mathrm{NE}}(B)=\{\langle a, b\rangle \in \mathbb{I} \times \mathbb{R} \mid$ there exists an open set $G$ such that $\langle a, b\rangle \in$ $G \&([0, a] \times \mathbb{R}) \cap G \subset \mathcal{N}(B) \&((a, 1] \times \mathbb{R}) \cap G \subset \mathcal{E}(B)\}$.

Theorem 1. Let $B \subset \mathbb{I} \times \mathbb{R}$ be a blocking set. Then:

1. $\{0\} \times \mathbb{R} \subset B \cup \mathcal{E}(B)$;

2. $\{1\} \times \mathbb{R} \subset B \cup \mathcal{N}(B)$;

3. $\mathcal{E}(B)$ is open; 
4. $\mathcal{N}(B)$ is left-open;

5. if for every open neighborhood $G$ of $\langle x, y\rangle$ there exist $\left\langle x_{1}, y_{1}\right\rangle \in G \cap \mathcal{E}(B)$, $\left\langle x_{2}, y_{2}\right\rangle \in G \cap \mathcal{N}(B)$ such that $x_{1}<x_{2}$, then $\langle x, y\rangle \in B$;

6. if $\langle x, y\rangle \notin B$ then $\langle x, y\rangle \in \mathrm{A}_{\mathrm{EE}}(B) \cup \mathrm{A}_{\mathrm{NN}}(B) \cup \mathrm{A}_{\mathrm{NE}}(B)$;

7. if $f: \mathbb{I} \rightarrow \mathbb{R}, f \in \mathrm{AC},\left\langle x_{1}, f\left(x_{1}\right)\right\rangle \in \mathcal{E}(B),\left\langle x_{2}, f\left(x_{2}\right)\right\rangle \in \mathcal{N}(B)$ and $x_{1}<x_{2}$, then there exists $x \in\left(x_{1}, x_{2}\right)$ such that $\langle x, f(x)\rangle \in B{ }^{1}$

Proof. (1), (2) These facts are easy consequences of definitions.

(3) Let $\langle p, q\rangle \in \mathcal{E}(B)$. Since $\langle p, q\rangle \notin B$, there exists a rectangular open neighborhood $G$ of $\langle p, q\rangle$ such that $G \cap B=\emptyset$. Let $h:[0, p] \rightarrow \mathbb{R}$ be continuous, $h \cap B=\emptyset$ and $h(p)=q$. There exists $\langle s, t\rangle \in \operatorname{bd}(G)$ such that $s<p$ and $h(s)=t$. For every point $\langle a, b\rangle$ from $((s, 1] \times \mathbb{R}) \cap G$ we can extend $h$ by linear segment $[\langle s, t\rangle,\langle a, b\rangle] \subset G$ to the continuous function $h^{\prime}:[0, a] \rightarrow \mathbb{R}$. Hence $\langle a, b\rangle \in \mathcal{E}(N)$, so $((s, 1] \times \mathbb{R}) \cap G \subset \mathcal{E}(B)$.

(4) Suppose $\langle p, q\rangle \in \mathcal{N}(B)$. Since $\langle p, q\rangle \notin B$, there exists a rectangular open neighborhood $G$ of $\langle p, q\rangle$ such that $G \cap B=\emptyset$. If $([0, p] \times \mathbb{R}) \cap G \not \subset \mathcal{N}(B)$ then there exists $\langle s, t\rangle \in G \cap \mathcal{E}(B)$ such that $s \leq p$. Because $\mathcal{E}(B)$ is open, we can assume that $s<p$. Let $h:[0, s] \rightarrow \mathbb{R}$ be a continuous function such that $h \cap B=\emptyset$ and $h(s)=t$. We can extend $h$ by the segment $[\langle s, t\rangle,\langle p, q\rangle] \subset G$ to the continuous function $h^{\prime}:[0, p] \rightarrow \mathbb{R}$ such that $h^{\prime} \cap B=\emptyset$, contrary to $\langle p, q\rangle \in \mathcal{N}(B)$.

(5) Suppose $\langle x, y\rangle \notin B$. Then there exists a rectangular open neighborhood $G$ of $\langle x, y\rangle$ such that $G \cap B=\emptyset$. Take $\left\langle x_{1}, y_{1}\right\rangle \in G \cap \mathcal{E}(B),\left\langle x_{2}, y_{2}\right\rangle \in G \cap \mathcal{N}(B)$, $x_{1}<x_{2}$. There exists continuous $h:\left[0, x_{1}\right] \rightarrow \mathbb{R}$ such that $h\left(x_{1}\right)=y_{1}$ and $h \cap B=\emptyset$. If we define $h^{\prime}:\left[0, x_{2}\right] \rightarrow \mathbb{R}$ as extension of $h$ by linear segment $\left[\left\langle x_{1}, y_{1}\right\rangle,\left\langle x_{2}, y_{2}\right\rangle\right] \subset G$ then $h^{\prime}$ is continuous. Since $\left\langle x_{2}, y_{2}\right\rangle \in \mathcal{N}(B)$, then $h^{\prime} \cap$ $B \neq \emptyset$, so $\left[\left\langle x_{1}, y_{1}\right\rangle,\left\langle x_{2}, y_{2}\right\rangle\right] \cap B \neq \emptyset$ and $G \cap B \neq \emptyset$. This is the contradiction.

(6) Suppose $\langle x, y\rangle \notin B$. Then either $\langle x, y\rangle \in \mathcal{E}(B)$ or $\langle x, y\rangle \in \mathcal{N}(B)$. If $\langle x, y\rangle \in \mathcal{E}(B)$ then, since $\mathcal{E}(B)$ is open, $\langle x, y\rangle \in \mathrm{A}_{\mathrm{EE}}(B)$. If $\langle x, y\rangle \in \mathcal{N}(B)$ then, since $\mathcal{N}(B)$ is left-open, there exists a rectangular open neighborhood $G$ of $\langle x, y\rangle$ such that $([0, x] \times \mathbb{R}) \cap G \subset \mathcal{N}(B)$ and $G \cap B=\emptyset$. Now suppose $\langle x, y\rangle \notin \mathrm{A}_{\mathrm{NN}}(B) \cup \mathrm{A}_{\mathrm{NE}}(B)$. This means that in every open neighborhood of $\langle x, y\rangle$ we can find points from $\mathcal{E}(B)$ and $\mathcal{N}(B)$ to the right of $x$. Take $\left\langle x_{2}, y_{2}\right\rangle \in G \cap \mathcal{N}(B), x_{2}>x$ and $\left\langle x_{1}, y_{1}\right\rangle \in\left(\left[0, x_{2}\right) \times \mathbb{R}\right) \cap G \cap \mathcal{E}(B)$. Now, analogously to (4), the linear segment $\left[\left\langle x_{1}, y_{1}\right\rangle,\left\langle x_{2}, y_{2}\right\rangle\right]$ intersects $B$, contrary to $G \cap B=\emptyset$.

\footnotetext{
${ }^{1}$ As an easy corollary we have that $f \in \mathrm{AC}$ iff for every blocking set $B$ and $\left\langle x_{1}, y_{1}\right\rangle \in$ $\mathcal{E}(B),\left\langle x_{2}, y_{2}\right\rangle \in \mathcal{N}(B)$ such that $x_{1}<x_{2}$ there exists $x \in\left(x_{1}, x_{2}\right)$ such that $\langle x, f(x)\rangle \in B$.
} 
(7) Let $\left\langle x_{1}, f\left(x_{1}\right)\right\rangle \in \mathcal{E}(B),\left\langle x_{2}, f\left(x_{2}\right)\right\rangle \in \mathcal{N}(B), x_{1}<x_{2}$. Let $h:\left[0, x_{1}\right] \rightarrow$ $\mathbb{R}$ be a continuous function such that $h \cap B=\emptyset$ and $h\left(x_{1}\right)=f\left(x_{1}\right)$. Suppose that for every $x \in\left(x_{1}, x_{2}\right),\langle x, f(x)\rangle \notin B$. So, for every $x \in\left[x_{1}, x_{2}\right]$, there exists open neighborhood $G_{\langle x, f(x)\rangle}$ of $\langle x, f(x)\rangle$ such that $G_{\langle x, f(x)\rangle} \cap B=\emptyset$. Let $G=\bigcup_{x \in\left[x_{1}, x_{2}\right]} G_{\langle x, f(x)\rangle}$. Since $f\left\lceil\left[x_{1}, x_{2}\right]\right.$ is almost continuous, there exists continuous function $g:\left[x_{1}, x_{2}\right] \rightarrow \mathbb{R}$ such that $g\left(x_{1}\right)=f\left(x_{1}\right), g\left(x_{2}\right)=f\left(x_{2}\right)$ and $g \subset G$. Since $G \cap B=\emptyset$, we can extend $h:\left[0, x_{1}\right] \rightarrow \mathbb{R}$ by $g$ to the continuous function $h^{\prime}:\left[0, x_{2}\right] \rightarrow \mathbb{R}$ such that $h^{\prime}\left(x_{2}\right)=f\left(x_{2}\right)$ and $h^{\prime} \cap B=\emptyset$. So $\left\langle x_{2}, f\left(x_{2}\right)\right\rangle$ belongs to $\mathcal{E}(B)$ rather than $\mathcal{N}(B)$. This is a contradiction.

Note that analogous facts remain true for functions from $\mathbb{I}$ to $\mathbb{I}$.

Remark 1. Let $B \subset \mathbb{I} \times \mathbb{R}$ be a blocking set. Suppose $\left\langle x, y_{1}\right\rangle \in \mathcal{E}(B)$ and $\left\langle x, y_{2}\right\rangle \in \mathcal{N}(B)$. Then there exists $y \in\left(y_{1}, y_{2}\right)$ such that $\langle x, y\rangle \in B$.

We will use Theorem 2 in the next part of the proof to analyze uniform limits of sequences of almost continuous functions.

Theorem 2. Let $f: \mathbb{I} \rightarrow \mathbb{R}$ be almost continuous and $B \subset \mathbb{I} \times \mathbb{R}$ be a blocking set. For every $a, b \in \mathbb{I}$, if $a<b,\langle a, f(a)\rangle \in \mathcal{E}(B)$ and $\langle b, f(b)\rangle \in \mathcal{N}(B)$ then at least one of the following statements holds:

1. there exists $x \in[a, b]$ and left or right-open neighborhood $U$ of $\langle x, f(x)\rangle$ such that $f \cap U \subset B$;

2. there exists $x \in[a, b]$ such that for every open neighborhood $V$ of $\langle x, f(x)\rangle$ there exists $x_{1}<x_{2}$ such that $\left\langle x_{1}, f\left(x_{1}\right)\right\rangle \in V \cap \mathcal{E}(B)$ and $\left\langle x_{2}, f\left(x_{2}\right)\right\rangle \in$ $V \cap \mathcal{N}(B)$.

(In the second case we will say that $f$ breaks through $B$ at $\langle x, f(x)\rangle$.)

Proof. Suppose, contrary to our claim, that neither (1) nor (2) hold. Denote:

$$
\begin{gathered}
B_{E E}=\left\{\langle x, f(x)\rangle \mid \text { there exists an open set } V_{\langle x, f(x)\rangle}\right. \text { such that } \\
\left.\langle x, f(x)\rangle \in V_{\langle x, f(x)\rangle} \& V_{\langle x, f(x)\rangle} \cap f \subset \mathcal{E}(B) \cup B\right\} \\
B_{N N}=\left\{\langle x, f(x)\rangle \mid \text { there exists an open set } V_{\langle x, f(x)\rangle}\right. \text { such that } \\
\left.\langle x, f(x)\rangle \in V_{\langle x, f(x)\rangle} \& V_{\langle x, f(x)\rangle} \cap f \subset \mathcal{N}(B) \cup B\right\}
\end{gathered}
$$

$B_{N E}=\left\{\langle x, f(x)\rangle \mid\right.$ there exists an open set $V_{\langle x, f(x)\rangle}$ such that

$$
\begin{aligned}
& \langle x, f(x)\rangle \in V_{\langle x, f(x)\rangle} \&([0, x] \times \mathbb{R}) \cap V_{\langle x, f(x)\rangle} \cap f \subset \mathcal{N}(B) \cup B \\
& \left.\&((x, 1] \times \mathbb{R}) \cap V_{\langle x, f(x)\rangle} \cap f \subset \mathcal{E}(B) \cup B\right\}
\end{aligned}
$$


First, note that sets $B_{E E} \cap((a, b) \times \mathbb{R}), B_{N N} \cap((a, b) \times \mathbb{R})$ and $B_{N E} \cap$ $((a, b) \times \mathbb{R})$ are pairwise disjoint. Indeed, suppose there exists $x \in(a, b)$ such that $\langle x, f(x)\rangle \in B_{E E} \cap B_{N E}$. So, there exists an open neighborhood $V_{\langle x, f(x)\rangle}$ of $\langle x, f(x)\rangle$ such that $([a, x] \times \mathbb{R}) \cap V_{\langle x, f(x)\rangle} \cap f \subset(\mathcal{E}(B) \cup B) \cap(\mathcal{N}(B) \cup B)=B$, contrary to the negation of (1). Analogously we prove that $B_{N N} \cap B_{N E}=\emptyset$ and $B_{E E} \cap B_{N N}=\emptyset$.

Next, note that $f \uparrow[a, b] \subset B_{E E} \cup B_{N N} \cup B_{N E}$. Indeed, it is easy to see that $\langle a, f(a)\rangle \in B_{E E}$ and $\langle b, f(b)\rangle \in B_{N N} \cup B_{N E}$. Now, take $x \in(a, b)$. There exists an open neighborhood $U$ of $\langle x, f(x)\rangle$ such that either $([a, x) \times \mathbb{R}) \cap U \cap$ $f \subset \mathcal{E}(B) \cup B$ or $([a, x) \times \mathbb{R}) \cap U \cap f \subset \mathcal{N}(B) \cup B$. (If it is not the case, since $([a, x) \times \mathbb{R}) \cap U \cap f \not \subset \mathcal{N}(B) \cup B$, we can find $x_{1}<x$ such that point $\left\langle x_{1}, f\left(x_{1}\right)\right\rangle \in \mathcal{E}(B) \cap U$. Next, since $\left(\left(x_{1}, x\right) \times \mathbb{R}\right) \cap U \cap f \not \subset \mathcal{E}(B) \cup B$, we find a point $\left\langle x_{2}, f\left(x_{2}\right)\right\rangle \in\left(\left(x_{1}, x\right) \times \mathbb{R}\right) \cap \mathcal{N}(B) \cap U$. We can find such points for arbitrary set $U$ - contrary to the negation of (2).) Using the same arguments we show that there exists an open neighborhood $V$ of $\langle x, f(x)\rangle$ such that either $((x, b) \times \mathbb{R}) \cap V \cap f \subset \mathcal{E}(B) \cup B$ or $((x, b) \times \mathbb{R}) \cap V \cap f \subset \mathcal{N}(B) \cup B$. Let $G=U \cap V$. Since $f$ is bilaterally dense in itself, the case $([a, x) \times \mathbb{R}) \cap G \cap f \subset \mathcal{E}(B) \cup B$ and $((x, b) \times \mathbb{R}) \cap G \cap f \subset \mathcal{N}(B) \cup B$ contradicts to the negation of (2). In any other case there exists an open neighborhood $V_{\langle x, f(x)\rangle}$ of $\langle x, f(x)\rangle$ as in the definition of $B_{E E}$ or $B_{N N}$ or $B_{N E}$. It is equivalent to $\langle x, f(x)\rangle \in B_{E E}$ or $\langle x, f(x)\rangle \in B_{N N}$ or $\langle x, f(x)\rangle \in B_{N E}$.

For $S_{\langle x, y\rangle}$ being an open square with the center $\langle x, y\rangle$ let $3 \cdot S_{\langle x, y\rangle}$ denote the open square with the center $\langle x, y\rangle$ and with the diagonal 3 times that of $S_{\langle x, y\rangle}$. For every $x \in[a, b]$ let $S_{\langle x, f(x)\rangle}$ be an open square with the center $\langle x, f(x)\rangle$ such that:

$$
\begin{aligned}
& 3 \cdot S_{\langle x, f(x)\rangle} \subset V_{\langle x, f(x)\rangle} \\
& 3 \cdot S_{\langle x, f(x)\rangle} \subset(a, b] \times \mathbb{R}, \text { for } x>a \\
& 3 \cdot S_{\langle x, f(x)\rangle} \subset[a, b) \times \mathbb{R}, \text { for } x<b
\end{aligned}
$$

Note that for $x>a$ the square $S_{\langle x, f(x)\rangle}$ does not contain points with abscissa $a$. Respectively, the square $S_{\langle x, f(x)\rangle}$ does not contain points with abscissa $b$ for $x<b$. Note also, that if $S_{\langle a, b\rangle} \cap S_{\langle c, d\rangle} \neq \emptyset$ then either $S_{\langle a, b\rangle} \subset$ $3 \cdot S_{\langle c, d\rangle}$ or $S_{\langle c, d\rangle} \subset 3 \cdot S_{\langle a, b\rangle} \cdot$

For every $x \in[a, b]$ let $R_{\langle x, f(x)\rangle}=\left(x_{l}, x_{r}\right) \times\left(y_{l}, y_{u}\right)$ be an open rectangular neighborhood of $\langle x, y\rangle$ such that $R_{\langle x, f(x)\rangle} \subset S_{\langle x, f(x)\rangle}, f\left(x_{l}\right) \in\left(y_{l}, y_{u}\right)$ for $x>a$ and $f\left(x_{r}\right) \in\left(y_{l}, y_{u}\right)$ for $x<b$.

Then for every $x \in[a, b]$ the set $R_{\langle x, f(x)\rangle}$ fulfills the following conditions:

$\left(o_{1}\right)$ if $\langle r, f(r)\rangle \in V_{\langle x, f(x)\rangle} \cap \mathcal{E}(B)$ for an $r \leq x$ then $\langle x, f(x)\rangle \in B_{E E}$ and for every $t>x_{l}$ there exists $z<t$ such that $\langle z, f(z)\rangle \in R_{\langle x, f(x)\rangle} \cap \mathcal{E}(B)$; 
$\left(\mathrm{o}_{2}\right)$ if $\langle r, f(r)\rangle \in V_{\langle x, f(x)\rangle} \cap \mathcal{E}(B)$ for an $r>x$ then $\langle x, f(x)\rangle \in B_{E E} \cup B_{N E}$ and for every $t>x$ there exists $z \in(x, t)$ such that $\langle z, f(z)\rangle \in R_{\langle x, f(x)\rangle} \cap$ $\mathcal{E}(B)$

$\left(\bullet_{1}\right)$ if $R_{\langle x, f(x)\rangle} \cap((r, x) \times \mathbb{R}) \cap f \subset \mathcal{E}(B) \cup B$ for an $r \in\left(x_{l}, x\right)$ then $\langle x, f(x)\rangle \in$ $B_{E E}$ and there exists $z \leq r$ such that $\langle z, f(z)\rangle \in R_{\langle x, f(x)\rangle} \cap \mathcal{E}(B)$;

$\left(\bullet_{2}\right)$ if $R_{\langle x, f(x)\rangle} \cap\left(\left(x, x_{r}\right) \times \mathbb{R}\right) \cap f \subset \mathcal{E}(B) \cup B$ for an $r \in\left(x, x_{r}\right)$ then $\langle x, f(x)\rangle \in$ $B_{E E} \cup B_{N E}$ and there exists $z \leq r$ such that $\langle z, f(z)\rangle \in R_{\langle x, f(x)\rangle} \cap \mathcal{E}(B)$.

Let $H=\bigcup_{x \in[a, b]} R_{\langle x, f(x)\rangle}$. $H$ is open and $f \subset H$, so there exists a continuous function $g:[a, b] \rightarrow \mathbb{R}$ such that $g \subset H$. Let $\mathcal{R}$ be a finite subfamily of $\left\{R_{\langle x, f(x)\rangle} \mid x \in[a, b]\right\}$ such that $g \subset \bigcup \mathcal{R}$.

Since only the $R_{\langle a, f(a)\rangle}$ contains points with abscissa $a$ and only the $R_{\langle b, f(b)\rangle}$ contains points with abscissa $b, R_{\langle a, f(a)\rangle} \in \mathcal{R}$ and $R_{\langle b, f(b)\rangle} \in \mathcal{R}$. Moreover, since $\mathcal{R}$ is finite and $3 \cdot S_{\langle x, f(x)\rangle} \subset([a, b) \times \mathbb{R})$ for every $x<b$,

$$
\sup \left\{x \in[a, b] \mid\langle x, y\rangle \in \bigcup\left(\mathcal{R} \backslash\left\{R_{\langle b, f(b)\rangle}\right\}\right)\right\}<b \text {. }
$$

Let $C=\left\{x \in[a, b] \mid(\exists R \in \mathcal{R})\left(\langle x, g(x)\rangle \in R \&\left(\exists x_{1} \leq x\right)\left\langle x_{1}, f\left(x_{1}\right)\right\rangle \in\right.\right.$ $\mathcal{E}(B) \cap R)\}$, and let $s=\sup C$. Since $\langle a, f(a)\rangle \in \mathcal{E}(B)$ and $\langle a, g(a)\rangle \in R_{\langle a, f(a)\rangle}$, there exists $x>a, x_{1} \in(a, x)$ such that $\langle x, g(x)\rangle \in R_{\langle a, f(a)\rangle},\left\langle x_{1}, f\left(x_{1}\right)\right\rangle \in$ $\mathcal{E}(B) \cap R_{\langle a, f(a)\rangle}$, so $s \geq x>a$. Analogously, the condition $(\star)$ implies $s<b$.

Since $\mathcal{R}$ is finite and $g$ is continuous, so there exists $R_{\langle p, f(p)\rangle} \in \mathcal{R}$ and $p_{1} \leq s$ such that $\langle s, g(s)\rangle \in \overline{R_{\langle p, f(p)\rangle}}$ and $\left\langle p_{1}, f\left(p_{1}\right)\right\rangle \in \mathcal{E}(B) \cap R_{\langle p, f(p)\rangle}$.

Let $R_{\langle q, f(q)\rangle} \in \mathcal{R}$ be an open rectangle such that $\langle s, g(s)\rangle \in R_{\langle q, f(q)\rangle}$. Since $\langle s, g(s)\rangle \in \overline{R_{\langle p, f(p)\rangle}} \cap R_{\langle q, f(q)\rangle}, R_{\langle p, f(p)\rangle} \cap R_{\langle q, f(q)\rangle} \neq \emptyset$.

We have two cases:

1. $R_{\langle p, f(p)\rangle} \subset 3 \cdot S_{\langle q, f(q)\rangle} \subset V_{\langle q, f(q)\rangle}$, if diameter of $S_{\langle p, f(p)\rangle}$ is less than diameter of $S_{\langle q, f(q)\rangle}$;

2. $R_{\langle q, f(q)\rangle} \subset 3 \cdot S_{\langle p, f(p)\rangle} \subset V_{\langle p, f(p)\rangle}$, otherwise.

Case 1. Then $\left\langle p_{1}, f\left(p_{1}\right)\right\rangle \in \mathcal{E}(B) \cap V_{\langle q, f(q)\rangle}$. There exists $q_{1} \leq s$ such that $\left\langle q_{1}, f\left(q_{1}\right)\right\rangle \in R_{\langle q, f(q)\rangle} \cap \mathcal{E}(B)$. Indeed, if $p_{1} \leq q$ then $\left\langle p_{1}, f\left(p_{1}\right)\right\rangle \in$ $([a, q] \times \mathbb{R}) \cap V_{\langle q, f(q)\rangle} \cap \mathcal{E}(B)$, so $\langle q, f(q)\rangle \in B_{E E}$ and there exists $q_{1} \leq s$ such that $\left\langle q_{1}, f\left(q_{1}\right)\right\rangle \in \mathcal{E}(B) \cap R_{\langle q, f(q)\rangle}$ (see the condition $\left(\circ_{1}\right)$ ). If $p_{1}>q$, then $\left\langle p_{1}, f\left(p_{1}\right)\right\rangle \in((q, b] \times \mathbb{R}) \cap V_{\langle q, f(q)\rangle} \cap \mathcal{E}(B)$ and $s>q$, so $\langle q, f(q)\rangle \in B_{E E} \cup B_{N E}$ and there exists $q_{1} \leq s$ such that $\left\langle q_{1}, f\left(q_{1}\right)\right\rangle \in \mathcal{E}(B) \cap R_{\langle q, f(q)\rangle}$ (see the condition $\left.\left(\mathrm{o}_{2}\right)\right)$.

Now, since $g$ is continuous and $s<b$, there exists $s_{1}>s$ such that $\left\langle s_{1}, g\left(s_{1}\right)\right\rangle \in R_{\langle q, f(q)\rangle}$, so $s_{1} \in C$, a contradiction. 
Case 2. There exists $q_{1}>s$ such that $\left\langle q_{1}, f\left(q_{1}\right)\right\rangle \in \mathcal{N}(B) \cap R_{\langle q, f(q)\rangle}$. Indeed, suppose by contradiction that no $q_{1}>s$ fulfills the claim. Since $g$ is continuous and $s<b$, there exists $s_{1}>s$ such that $\left\langle s_{1}, g\left(s_{1}\right)\right\rangle \in R_{\langle q, f(q)\rangle}$ and $s_{1} \neq q$. By supposition, $\left(\left(s_{1}, b\right] \times \mathbb{R}\right) \cap R_{\langle q, f(q)\rangle} \cap \mathcal{N}(B) \cap f=\emptyset$, so $\left(\left(s_{1}, b\right] \times \mathbb{R}\right) \cap R_{\langle q, f(q)\rangle} \cap f \subset \mathcal{E}(B) \cup B$. If $s_{1}<q$ then there exists $z \leq s_{1}$ such that $\langle z, f(z)\rangle \in \mathcal{E}(B) \cap R_{\langle q, f(q)\rangle}$ (see the condition $\left.\left(\bullet_{1}\right)\right)$. If $s_{1}>q$ then there exists $z \leq s_{1}$ such that $\langle z, f(z)\rangle \in \mathcal{E}(B) \cap R_{\langle q, f(q)\rangle}$ (see the condition $\left(\bullet_{2}\right)$ ). In any case, $s_{1} \in C$. Since this is a contradiction, there exists $q_{1}>s$ such that $\left\langle q_{1}, f\left(q_{1}\right)\right\rangle \in \mathcal{N}(B) \cap R_{\langle q, f(q)\rangle}$.

Since $\left\langle q_{1}, f\left(q_{1}\right)\right\rangle \in \mathcal{N}(B) \cap R_{\langle q, f(q)\rangle}$, so $\left\langle q_{1}, f\left(q_{1}\right)\right\rangle \in \mathcal{N}(B) \cap V_{\langle p, f(p)\rangle}$. But $\left\langle p_{1}, f\left(p_{1}\right)\right\rangle \in \mathcal{E}(B) \cap V_{\langle p, f(p)\rangle}$ and $p_{1}<q_{1}$-it is impossible, since $\langle p, f(p)\rangle \in$ $B_{E E} \cup B_{N N} \cup B_{N E}$.

\section{Some new proofs of old theorems and a proof of the new one}

We will show below that some classical theorems can be proved in a shorter way by using the theory of operators $\mathcal{E}(\cdot)$ and $\mathcal{N}(\cdot)$.

Theorem 3 ([1]). Every $\mathrm{DB}_{1}$ function $f: \mathbb{I} \rightarrow \mathbb{R}$ is almost continuous.

Proof. Suppose $f: \mathbb{I} \rightarrow \mathbb{R}$ is $\mathrm{DB}_{1}$ and there exists blocking set $B \subset \mathbb{I} \times \mathbb{R}$ such that $f \subset \mathcal{E}(B) \cup \mathcal{N}(B)$. Recall that $f$, as a Darboux function, is bilaterally dense in itself.

Denote:

$$
\begin{aligned}
E^{\prime} & =\{x \in \mathbb{I} \mid\langle x, f(x)\rangle \in \mathcal{E}(B)\} \\
N^{\prime} & =\{x \in \mathbb{I} \mid\langle x, f(x)\rangle \in \mathcal{N}(B)\}
\end{aligned}
$$

By Theorem 1, we have $E^{\prime} \cap N^{\prime}=\emptyset, 0 \in E^{\prime}, 1 \in N^{\prime}$ and $E^{\prime} \cup N^{\prime}=\mathbb{I}$. Let $K$ be the set of all $x \in \mathbb{I}$ such that for every open neighborhood $G$ of $x$ there exist $x_{1}, x_{2} \in G$ with $x_{1}<x_{2}$ and $x_{1} \in E^{\prime}, x_{2} \in N^{\prime}$.

Note that for $x_{1}<x_{2}, x_{1} \in E^{\prime}, x_{2} \in N^{\prime}$ there exists $x \in\left[x_{1}, x_{2}\right]$ such that $x \in K\left(\right.$ E. g. $\left.\sup \left(E^{\prime} \cap\left[x_{1}, x_{2}\right]\right) \in K\right)$, so $K \neq \emptyset$. It is also easy to see that $K$ is closed. Since $f$ is Baire class 1 , there exists $p \in K$ such that $f \uparrow K$ is continuous at $p$. Since $\langle p, f(p)\rangle \notin B,\langle p, f(p)\rangle \in \mathrm{A}_{\mathrm{NE}}(B) \cup \mathrm{A}_{\mathrm{NN}}(B) \cup \mathrm{A}_{\mathrm{EE}}(B)$. During the rest of the proof we will suppose $\langle p, f(p)\rangle \in \mathrm{A}_{\mathrm{NE}}(B)$; the proof in other cases is analogous.

Since $f\lceil K$ is continuous at $p$, there exists an open neighborhood $G$ of $p$ such that $[0, p] \cap G \cap K \subset N^{\prime}$ and $(p, 1] \cap G \cap K \subset E^{\prime}$. We will show that $[0, p] \cap G \subset N^{\prime}$ and $(p, 1] \cap G \subset E^{\prime}$. 
Indeed, suppose there exists $p_{1}<p$ such that $p_{1} \in G \cap E^{\prime}$. Then $p_{1} \notin K$, so $p_{1}$ is contained in a component $(a, b)$ of $\mathbb{I} \backslash K$. Since $b \in K$ and $b \leq p, b \in N^{\prime}$. Since $\langle b, f(b)\rangle \in \mathcal{N}(B), \mathcal{N}(B)$ is left-open and $f$ is bilaterally dense in itself, there exists $b_{1} \in\left(p_{1}, b\right)$ such that $b_{1} \in N^{\prime}$. But now there exists $k \in K$ such that $p_{1} \leq k \leq b_{1}$, contrary to $\left[p_{1}, b_{1}\right] \subset(a, b) \subset \mathbb{I} \backslash K$. Analogous arguments work for the right side of $p$.

Since $[0, p] \cap G \subset N^{\prime}$ and $(p, 1] \cap G \subset E^{\prime}, p \notin K$. This is a contradiction.

Theorem 4 ([2]). The Cesáro-Vietoris function $\phi: \mathbb{I} \rightarrow \mathbb{R}$ defined by:

$$
\phi(x)=\limsup _{n \rightarrow \infty} \frac{a_{1}+a_{2}+\cdots+a_{n}}{n}
$$

(where the $a_{i}$ are given by the unique nonterminating binary expansion of $x$ ) is almost continuous.

Proof. Suppose there exists a blocking set $B \subset \mathbb{I} \times \mathbb{I}$, such that $\phi \subset \mathcal{E}(B) \cup$ $\mathcal{N}(B)$.

First, observe that $\phi$ is Darboux. Let $s=\sup \{x \mid\langle x, \phi(x)\rangle \in \mathcal{E}(B)\}$. Since $\langle 0, \phi(0)\rangle \in \mathcal{E}(B), \mathcal{E}(B)$ is open and $\phi$ is bilaterally dense in itself, so $s>0$ and $\langle s, \phi(s)\rangle \in \mathcal{N}(B)$. By theorem 1 (4) there are $a, b$ and $c$ such that $a<s, b<\phi(s)<c$ and $(a, s) \times(b, c) \subset \mathcal{N}(B)$. Let $s^{\prime} \in(a, s)$ be such that $\left\langle s^{\prime}, \phi\left(s^{\prime}\right)\right\rangle \in \mathcal{E}(B)$. Take $a^{\prime}, b^{\prime}$ and $c^{\prime}$ such that $s^{\prime}<a^{\prime}<s, b^{\prime}<\phi\left(s^{\prime}\right)<c^{\prime}$ and $\left(s^{\prime}, a^{\prime}\right) \times\left(b^{\prime}, c^{\prime}\right) \subset \mathcal{E}(B)$. Then we have the vertical strip $W=\left[s^{\prime}, a^{\prime}\right] \times \mathbb{R}$ such that every vertical line lying in $W$ contain a point of $\mathcal{E}(B)$, a point of $\mathcal{N}(B)$ and therefore a point of $B$. (See Remark 1.)

The rest of the proof is a modification of Brown's (Vietoris) proof. Let $B_{0}=B \cap W$ and $\left\{W_{n}\right\}_{n}$ be a descending sequence of open neighborhoods of $B_{0}$ such that $B_{0}=\bigcap_{n \in \mathbb{N}} W_{n}$. We will define an ascending sequence $n_{1}, n_{2}, \ldots$ of natural numbers and dyadic decimal $0 . a_{1} a_{2} \cdots a_{n_{1}} a_{n_{1}+1} \cdots a_{n_{2}} a_{n_{2}+1} \cdots$ simultaneously.

Take any dyadic rational $\xi_{0}=0 . a_{1} a_{2} \cdots a_{i} \in\left(s^{\prime}, a^{\prime}\right)$ and $\eta_{0}>0$ such that $\left\langle\xi_{0}, \eta_{0}\right\rangle \in W_{1} \cap W$. Define $Q$ as a square neighborhood of $\left\langle\xi_{0}, \eta_{0}\right\rangle$ which has radius $q<\frac{\eta_{0}}{2}$ and lies interior to $W_{1} \cap W$. Now, take $k>i$ such that if we put $a_{i+1}=a_{i+2}=\cdots=a_{k}=0$, then:

1. $\xi_{0}<0 . a_{1} a_{2} \cdots a_{k} 111 \cdots<\xi_{0}+q$; and

2. $M_{k}<q$, with $M_{k}$ denoting $\frac{a_{1}+a_{2}+\cdots a_{k}}{k}$; and

3. $\frac{1}{k}<q$.

Condition 1 implies that regardless of how $0 . a_{1} a_{2} \cdots a_{k}$ is continued it will differ from $\xi_{0}$ by less than $q$. Then define $a_{k+1}=a_{k+2}=\cdots=a_{n_{1}}=1$, such 
that $\left\langle\xi_{1}, M_{n_{1}}\right\rangle \in Q$, with $\xi_{1}$ denoting $0 . a_{1} a_{2} \cdots a_{n_{1}}$. (This can be accomplished since $\left|M_{i+1}-M_{i}\right|<\frac{1}{i+1}<q$ for each $i>k$.)

If we have defined $\xi_{m}=0 . a_{1} a_{2} \cdots a_{n_{m}} \in\left(s^{\prime}, a^{\prime}\right)$ such that $\left\langle\xi_{m}, M_{n_{m}}\right\rangle \in$ $W_{m} \cap W$, we can repeat the process, starting from the point $\left\langle\xi_{m}, \eta_{m}\right\rangle$ of $W_{m+1} \cap$ $W$ with $\eta_{m}>0$, and define $a_{n_{m}+1}, a_{n_{m}+2}, \ldots, a_{n_{m+1}}$ first using consecutive 0 's and then using consecutive 1's, so that $\left\langle\xi_{m+1}, M_{n_{m+1}}\right\rangle \in W_{m+1} \cap W$.

Define $\xi_{\omega}=0 . a_{1} a_{2} \cdots$. Since consecutive 0's and then consecutive 1's were used in proceeding from $a_{n_{r}}$ to $a_{n_{r+1}}$ in the above induction, for each $i$ between $n_{r}$ and $n_{r+1}$ we have $M_{i} \leq \max \left\{M_{n_{r}}, M_{n_{r+1}}\right\}$, so $\phi\left(\xi_{\omega}\right)=\lim \sup _{i \rightarrow \infty} M_{i}=$ $\lim \sup _{r \rightarrow \infty} M_{n_{r}}$. Therefore, the point $\left\langle\xi_{\omega}, \phi\left(\xi_{\omega}\right)\right\rangle$ is the limit of some subsequence $\left\langle\xi_{m_{r}}, M_{n_{m_{r}}}\right\rangle$ of $\left\langle\xi_{m}, M_{n_{m}}\right\rangle$. Since $\left\langle\xi_{m_{r}}, M_{n_{m_{r}}}\right\rangle \in W_{m_{r}}$ and $\bigcap_{r \in \mathbb{N}} W_{m_{r}}=$ $B_{0},\left\langle\xi_{\omega}, \phi\left(\xi_{\omega}\right)\right\rangle \in B_{0} \subset B$, contrary to $\phi \cap B=\emptyset$.

The next theorem is connected with a problem: "Given a Darboux function $f: \mathbb{I} \rightarrow \mathbb{I}$, does there exists almost continuous function $g: \mathbb{I} \rightarrow \mathbb{I}$ with $g \subset \bar{f}$ ?" (see [10]). This problem has been solved by H. Rosen in more general case of extendible functions (see [13]). Here we will present an easier and shorter proof for the case of almost continuous function.

Theorem 5. For every Darboux function $f: \mathbb{I} \rightarrow \mathbb{I}$ there exists almost continuous function $g: \mathbb{I} \rightarrow \mathbb{I}$ with $g \subset \bar{f}$.

Proof. Let:

$$
\begin{aligned}
& C_{-}\left(x_{0}\right)=\left\{y \in \mathbb{I} \mid \text { there exists a sequence }\left\{x_{n}\right\}_{n} \subset\left[0, x_{0}\right], f\left(x_{n}\right) \rightarrow y\right\} \\
& C_{+}\left(x_{0}\right)=\left\{y \in \mathbb{I} \mid \text { there exists a sequence }\left\{x_{n}\right\}_{n} \subset\left[x_{0}, 1\right], f\left(x_{n}\right) \rightarrow y\right\}
\end{aligned}
$$

It is easy to see that $\bar{f}=\bigcup_{x \in \mathbb{I}}\left(\{x\} \times\left(C_{-}(x) \cup C_{+}(x)\right)\right)$. It is also known that for every Darboux function $f$ and $x \in \mathbb{I}, f$ is bilaterally $\mathfrak{c}$-dense in itself, sets $C_{-}(x)$ and $C_{+}(x)$ are closed intervals and by well-known Young theorem, set $\left\{x \in \mathbb{I} \mid C_{-}(x) \neq C_{+}(x)\right\}$ is countable.

Now we will show that for every blocking set $B$ at least one of the following conditions holds:

1. there exists $x \in \mathbb{I}$ such that $C_{-}(x) \cap C_{+}(x) \subset B_{x}$;

2. the set $\left\{x \in \mathbb{I} \mid\left(C_{-}(x) \cup C_{+}(x)\right) \cap B_{x} \neq \emptyset\right\}$ has cardinality $\mathfrak{c}$.

Suppose that there exists blocking set $B$ such that neither (1) nor (2) holds. Then for every $x \in \mathbb{I}$ there exists $y \in\left(C_{-}(x) \cap C_{+}(x)\right) \backslash B_{x}$ and the set $\{x \in \mathbb{I} \mid\langle x, f(x)\rangle \in B\}$ has cardinality less than $\mathfrak{c}$.

Denote:

$$
E^{\prime}=\{x \in \mathbb{I} \mid\langle x, f(x)\rangle \in \mathcal{E}(B)\}
$$




$$
\begin{aligned}
N^{\prime} & =\{x \in \mathbb{I} \mid\langle x, f(x)\rangle \in \mathcal{N}(B)\} \\
B^{\prime} & =\{x \in \mathbb{I} \mid\langle x, f(x)\rangle \in B\}
\end{aligned}
$$

Sets $E^{\prime}, N^{\prime}$ and $B^{\prime}$ are pairwise disjoint, $0 \in E^{\prime} \cup B^{\prime}, 1 \in N^{\prime} \cup B^{\prime}$ and $E^{\prime} \cup N^{\prime} \cup B^{\prime}=\mathbb{I}$.

Let $K$ be the set of all $x \in \mathbb{I}$ such that for every open neighborhood $G$ of $x$ there exists $x_{1}, x_{2} \in G$ with $x_{1}<x_{2}, x_{1} \in E^{\prime}$ and $x_{2} \in N^{\prime}$. It is easy to see that $K$ is closed.

Note that $K \subset\left\{x \in \mathbb{I} \mid\left(C_{-}(x) \cup C_{+}(x)\right) \cap B_{x} \neq \emptyset\right\}$. Indeed, for every open neighborhood $G$ of $x \in K$, if we take $x_{1}<x_{2},\left\langle x_{1}, f\left(x_{1}\right)\right\rangle \in \mathcal{E}(B)$ and $\left\langle x_{2}, f\left(x_{2}\right)\right\rangle \in \mathcal{N}(B)$, then (by a slight change in the proof of Theorem $1(5)$ ) there exists $x_{3} \in\left(x_{1}, x_{2}\right)$ and $y_{3} \in\left(f\left(x_{1}\right), f\left(x_{2}\right)\right)$ (we can assume that $f\left(x_{1}\right)<$ $\left.f\left(x_{2}\right)\right)$ such that $\left\langle x_{3}, y_{3}\right\rangle \in B$. Having sequences $\left\{\left\langle x_{1}^{n}, f\left(x_{1}^{n}\right)\right\rangle\right\}_{n} \subset \mathcal{E}(B), x_{1}^{n} \rightarrow$ $x$ and $\left\{\left\langle x_{2}^{n}, f\left(x_{2}^{n}\right)\right\rangle\right\}_{n} \subset \mathcal{N}(B), x_{2}^{n} \rightarrow x$, we can build a sequence $\left\{\left\langle x_{3}^{n}, y_{3}^{n}\right\rangle\right\}_{n} \subset$ $B$ such that $\left|y_{3}^{n}-f(x)\right| \leq \max \left\{\left|f\left(x_{1}^{n}\right)-f(x)\right|,\left|f\left(x_{2}^{n}\right)-f(x)\right|\right\}$. Since $B$ is compact and $\left\{\left\langle x_{3}^{n}, y_{3}^{n}\right\rangle\right\}_{n} \subset B$ then there exists a subsequence of $\left\{\left\langle x_{3}^{n}, y_{3}^{n}\right\rangle\right\}_{n}$ which converges to some $\langle x, y\rangle \in B$. Thus $y \in\left(C_{-}(x) \cup C_{+}(x)\right) \cap B_{x}$.

Note also that for $x_{1}<x_{2}, x_{1} \in E^{\prime}, x_{2} \in N^{\prime}$ there exists $x \in\left[x_{1}, x_{2}\right]$ such that $x \in K$. Indeed, $x=\sup \left(E^{\prime} \cap\left[x_{1}, x_{2}\right]\right) \in K$. Since $f$ is bilaterally dense in itself, $\langle x, f(x)\rangle \in B \cup \mathcal{N}(B)$. If $x \in B^{\prime}$, then $\left(x, x_{2}\right] \cap N^{\prime}$ is dense in $x$ (the cardinality of $B^{\prime}$ is less then $\mathfrak{c}$ ). If $x \in N^{\prime}$ then $\left[x_{1}, x\right) \cap N^{\prime}$ is dense in $x$ ( $f$ is left side dense in itself and $\mathcal{N}(B)$ is left-open). As a corollary we have $K \neq \emptyset$.

Now, we will show that $K$ is dense in itself. Let $x \in K$. There exists $y \in\left(C_{-}(x) \cap C_{+}(x)\right) \backslash B_{x}$, so by Theorem $1(6)\langle x, y\rangle \in \mathrm{A}_{\mathrm{EE}}(B) \cup \mathrm{A}_{\mathrm{NN}}(B) \cup$ $\mathrm{A}_{\mathrm{NE}}(B)$. The proof for these three cases is analogous, so suppose $\langle x, y\rangle \in$ $\mathrm{A}_{\mathrm{NE}}(B)$. Take an open rectangular neighborhood $(a, b) \times(c, d)$ of $\langle x, y\rangle$ such that $(a, x) \times(c, d) \subset \mathcal{N}(B)$ and $(x, b) \times(c, d) \subset \mathcal{E}(B)$. Since $x \in K$, there exists $x_{1} \in(a, b) \cap E^{\prime}$ and $x_{2} \in(a, b) \cap N^{\prime}, x_{1}<x_{2}$. Either $x_{1}<x$ or $x_{2}>x$.

If $x_{1}<x$ then $\left\langle x_{1}, f\left(x_{1}\right)\right\rangle \in \mathcal{E}(B)$. Since $y \in C_{-}(x)$ and $\langle x, y\rangle \in$ $\mathrm{A}_{\mathrm{NE}}(B)$, there exists $x^{\prime} \in\left(x_{1}, x\right)$ such that $\left\langle x^{\prime}, f\left(x^{\prime}\right)\right\rangle \in \mathcal{N}(B)$. Now we have $\left\langle x_{1}, f\left(x_{1}\right)\right\rangle \in \mathcal{E}(B),\left\langle x^{\prime}, f\left(x^{\prime}\right)\right\rangle \in \mathcal{N}(B)$ and $x_{1}<x^{\prime}$, so there exists $x^{\prime \prime} \in\left[x_{1}, x^{\prime}\right] \cap K$.

Analogously, if $x_{2}>x$ then we can find $x^{\prime} \in\left(x, x_{2}\right)$ such that $\left\langle x^{\prime}, f\left(x^{\prime}\right)\right\rangle \in$ $\mathcal{E}(B)$, so there exists $x^{\prime \prime} \in\left[x^{\prime}, x_{2}\right] \cap K$.

So, $K \subset\left\{x \in \mathbb{I} \mid\left(C_{-}(x) \cup C_{+}(x)\right) \cap B_{x} \neq \emptyset\right\}$. But $K$ is closed and dense in itself, so $K$ has cardinality $c$. This is a contradiction with the negation of condition (2).

Now we can construct by transfinite induction a function $g$ such that $g \subset \bar{f}$ and $g \cap B \neq \emptyset$ for every blocking set $B$. Let $A=\left\{x \in \mathbb{I} \mid C_{-}(x) \neq C_{+}(x)\right\}$. (By Young theorem, $A$ is countable.) Let $\left\{B_{\alpha}\right\}_{\alpha<\mathfrak{c}}$ be an ordered family of all blocking sets $B$ such that the set $\left\{x \in \mathbb{I} \mid\left(C_{-}(x) \cup C_{+}(x)\right) \cap B_{x} \neq \emptyset\right\}$ 
has cardinality $\mathfrak{c}$. For every $\alpha<\mathfrak{c}$ choose $\left\langle x_{\alpha}, y_{\alpha}\right\rangle \in B_{\alpha}$ such that $x_{\alpha} \in$ $\mathbb{I} \backslash\left(A \cup\left\{x_{\beta} \mid \beta<\alpha\right\}\right)$ and $y_{\alpha} \in C_{-}\left(x_{\alpha}\right) \cap C_{+}\left(x_{\alpha}\right)$. Define $g$ by

$$
g(x)= \begin{cases}y_{\alpha} & \text { if } x=x_{\alpha} \text { for an } \alpha<\mathfrak{c} \\ y \in C_{-}(x) \cap C_{+}(x) & \text { otherwise }\end{cases}
$$

It is easy to see that $g \subset \bigcup_{x \in \mathbb{I}}\left(\{x\} \times\left(C_{-}(x) \cap C_{+}(x)\right)\right) \subset \bar{f}$ and $g$ is almost continuous.

It is known, that if $f: \mathbb{I} \rightarrow \mathbb{R}$ is almost continuous, then $\max (f, g)$ is almost continuous for every Darboux upper semicontinuous function $g: \mathbb{I} \rightarrow \mathbb{R}([9])$. We don't know if the converse statement is true, but we can prove the following fact.

Theorem 6. Suppose $f: \mathbb{I} \rightarrow \mathbb{R}$. If $\max (f, c) \in \mathrm{AC}$ and $\min (f, c) \in \mathrm{AC}$ for every real number $c$, then $f \in \mathrm{AC}$.

Proof. Suppose, $\max (f, c) \in \mathrm{AC}$ and $\min (f, c) \in \mathrm{AC}$ for every $c \in \mathbb{R}$ and there exists a blocking set $B$ with $B \cap f=\emptyset$. Let

$$
s=\sup \{x \in \mathbb{I} \mid\langle x, f(x)\rangle \in \mathcal{E}(B)\} .
$$

Then either $\langle s, f(s)\rangle \in \mathcal{E}(B)$ or $\langle s, f(s)\rangle \in \mathcal{N}(B)$.

If $\langle s, f(s)\rangle \in \mathcal{E}(B)$ then $s<1$ and there exists a rectangular right-open neighborhood $\left[s, x_{1}\right) \times\left(y_{1}, y_{2}\right) \subset \mathcal{E}(B)$ of $\langle s, f(s)\rangle$. Fix $t \in\left(s, x_{1}\right)$ such that $\langle t, f(t)\rangle \in \mathcal{N}(B)$. Suppose $f(t)<f(s)(f(t)>f(s))$ and consider $g=$ $\min (f, f(s))(g=\max (f, f(s)))$. Since $g \in \mathrm{AC}$ and $\langle s, g(s)\rangle=\langle s, f(s)\rangle \in \mathcal{E}(B)$ and $\langle t, g(t)\rangle=\langle t, f(t)\rangle \in \mathcal{N}(B)$, by Theorem 1 (7) there exists $x \in(s, t)$ such that $\langle x, g(x)\rangle \in B$. This is the contradiction, because $g(x) \neq f(x)$ (since $f \cap B=\emptyset)$ and $g(x) \neq f(s)$ (since $\langle x, f(s)\rangle \in\left[s, x_{1}\right) \times\left(y_{1}, y_{2}\right) \subset \mathcal{E}(B)$ ).

The case $\langle s, f(s)\rangle \in \mathcal{N}(B)$ is analogous if we take into consideration leftopen rectangular neighborhood of $\langle s, f(s)\rangle$ contained in $\mathcal{N}(B)$.

Note that in the above proof we use only the following property of the family $C$ of constant functions: for every $\langle x, y\rangle \in \mathbb{I} \times \mathbb{R}$ there exists right-open (left-open) set $G=\left[x, x_{2}\right) \times\left(y_{1}, y_{2}\right)\left(G=\left(x_{1}, x\right] \times\left(y_{1}, y_{2}\right)\right)$ and a function $c \in C$ such that $\langle x, y\rangle \in G$ and $c\left\lceil\left[x, x_{2}\right) \subset G\left(c\left\lceil\left(x_{1}, x\right] \subset G\right)\right.\right.$. So, we can formulate the general corollary.

Corollary 1. Suppose $C$ is a subfamily of continuous real functions such that $\bigcup C$ is dense in the plane. If $f: \mathbb{I} \rightarrow \mathbb{R}$ is such that $\max (f, g) \in \mathrm{AC}$ and $\min (f, g) \in \mathrm{AC}$ for every $g \in C$, then $f$ is almost continuous.

Note that, in particular, $C$ can be countable. 


\section{Uniform limits of almost continuous functions}

The problem of characterization of uniform limits of AC functions has been posed by K. Kellum in [7]. This problem remains still open ([5]) and it seems to be the most interesting unsolved problem concerning almost continuous functions. We will apply Theorem 2 to obtain some partial results concerning this problem.

First, recall two notions defined by Bruckner, Ceder and Weiss.

Definition 3. Let $f: \mathbb{I} \rightarrow \mathbb{R}$.

- $f$ is in class $\mathcal{U}_{0}$ if for every interval $J \subset \mathbb{I}$ the set $f(J)$ is dense in the interval $\left[\inf _{x \in J} f(x), \sup _{x \in J} f(x)\right]$;

- $f$ is in class $\mathcal{U}$ if for every interval $J \subset \mathbb{I}$ and every set $A \subset \mathbb{I}$ such that cardinality of $A$ is less than $\mathfrak{c}$ the set $f(J \backslash A)$ is dense in the interval $\left[\inf _{x \in J} f(x), \sup _{x \in J} f(x)\right]$.

Note that $\overline{\mathrm{D}}=\mathcal{U}([3])$.

Of course, $\overline{\mathrm{AC}} \subset \mathcal{U}$. An example showing that $\overline{\mathrm{AC}} \neq \mathcal{U}$ was found by Kellum ([7], see also [6]). The next definition is connected with necessary and sufficient conditions for a function $f: \mathbb{I} \rightarrow \mathbb{R}$ to be in $\overline{\mathrm{AC}}$.

Definition 4. Let $B$ be a blocking set and $\varepsilon>0$.

- $\alpha(B, \varepsilon)$ is the class of all $f: \mathbb{I} \rightarrow \mathbb{R}$ for which at least one of the following conditions holds:

1. the set $\left\{x \in \mathbb{I}|| f(x)-B_{x} \mid<\varepsilon\right\}$ has cardinality $\mathfrak{c}$;

2. there exists $x \in \mathbb{I}$ such that $[f(x)-\varepsilon, f(x)+\varepsilon] \subset B_{x}$.

- $\beta(B, \varepsilon)$ is the class of all $f: \mathbb{I} \rightarrow \mathbb{R}$ for which at least one of the following conditions holds:

1. the set $\left\{x \in \mathbb{I}|| f(x)-B_{x} \mid<\varepsilon\right\}$ has cardinality $\mathfrak{c}$;

2. there exists $x \in \mathbb{I}$ such that $[f(x)-\varepsilon, f(x)+\varepsilon] \cap B_{x}$ has non-empty interior.

We say that $f$ is in class $\alpha$ (respectively $f$ is in $\beta$ ) iff $f \in \alpha(B, \varepsilon)$ (respectively $f \in \beta(B, \varepsilon)$ ) for every blocking set $B$ and $\varepsilon>0$.

T. Natkaniec proved in [11] that $\alpha \subset \overline{\mathrm{AC}}$ and, under $\mathrm{CH}, \overline{\mathrm{AC}} \subset \beta$ (we will show later that $\bar{\alpha}=\overline{\mathrm{AC}} \subset \beta$ in $\mathrm{ZFC})^{2}$. He also proved the following theorem.

\footnotetext{
${ }^{2}$ The definition of the class $\alpha$ given by Natkaniec is slightly weaker than used in this paper, since for a function $f$ to belong to $\alpha$ he requires $f \in \alpha(B, \varepsilon)$ for sufficiently small $\varepsilon>0$ and every blocking set $B$. Author does not know if both definitions are equivalent.
} 
Theorem 7. Let $f: \mathbb{I} \rightarrow \mathbb{R}$ and $\varepsilon>0$. Suppose that $\{x \in \mathbb{I}|| f(x)-q \mid \leq \varepsilon\}$ is $\mathfrak{c}$-dense in $\mathbb{I}$ for every rational $q$. Then $f$ fulfills the first condition from the definition of the class $\alpha(B, \varepsilon)$.

In the particular, if $f \in \mathcal{U}=\overline{\mathrm{D}}$ and the graph of $f$ is dense in the plane, then $f \in \overline{\mathrm{AC}}$.

The first from the following definitions belongs to Kellum ${ }^{3}$ :

Definition 5. Let $f: \mathbb{I} \rightarrow \mathbb{R}$.

- $f \in \mathrm{AAC}_{0}$ ( $f$ is away-almost continuous) iff $\left\{x|| f(x)-B_{x} \mid<\varepsilon\right\}$ is non-empty for every blocking set $B$ and $\varepsilon>0$;

- $f \in$ AAC iff $f \cap B \neq \emptyset$ or $\left\{x|| f(x)-B_{x} \mid<\varepsilon\right\}$ has cardinality $\mathfrak{c}$ for every blocking set $B$ and $\varepsilon>0$.

Obviously $\mathrm{AAC} \subset \mathrm{AAC}_{0}$. It is also easy to see that $\overline{\mathrm{AC}} \subset \mathrm{AAC}_{0}$.

During Miniconference in Real Analysis in Auburn in 1999 Kellum formulated conjecture that $f$ is in $\overline{\mathrm{AC}}$ iff $f \in \mathcal{U} \cap \mathrm{AAC}_{0}$.

Note the alternate definition of AAC.

Remark 2. A function $f$ is in AAC iff for every $\varepsilon>0$, every set $J \subset$ $\mathbb{I}$ of cardinality less than $\mathfrak{c}$ and every open neighbourhood $G$ of the set $f \cup$ $\bigcup_{x \in \mathbb{I} \backslash J}(\{x\} \times[f(x)-\varepsilon, f(x)+\varepsilon])$ there exists a continuous function $g: \mathbb{I} \rightarrow$ $\mathbb{R}$ such that $g \subset G$.

\section{Theorem 8.}

1. $\mathrm{AC} \subset \alpha$;

2. $\bar{\alpha} \subset \beta \cap \mathrm{AAC}$;

3. $\mathrm{AAC} \subset \mathcal{U} \cap \mathrm{AAC}_{0}$.

Lemma 1. Suppose $f: \mathbb{I} \rightarrow \mathbb{R}$ is bilaterally $\mathfrak{c}$-dense in itself, $c \in \mathbb{I}$ and either

1. $\langle c, f(c)\rangle \in \mathcal{E}(B)$ and $\left|f(c)-\mathcal{N}(B)_{c}\right|<\varepsilon$, or

2. $\langle c, f(c)\rangle \in \mathcal{N}(B)$ and $\left|f(c)-\mathcal{E}(B)_{c}\right|<\varepsilon$.

Then the set $\left\{x \in \mathbb{I}|| f(x)-B_{x} \mid<\varepsilon\right\}$ has cardinality $\mathfrak{c}$.

\footnotetext{
${ }^{3}$ Originally the class $\mathrm{AAC}_{0}$ was called $\mathrm{AAC}$. We decide to change its name to $\mathrm{AAC}_{0}$ to obtain duality with classes $\mathcal{U}$ and $\mathcal{U}_{0}$.
} 
Proof. We will prove this lemma only for the case (1).

Take $y \in \mathbb{R}$ such that $\langle c, y\rangle \in \mathcal{N}(B)$ and $|y-f(c)|<\varepsilon$. By Theorem 1 (3) and (4), there exists $\tau>0$ such that $(c-\tau, c+\tau) \times\left(f(c)-\frac{\tau}{2}, f(c)+\frac{\tau}{2}\right) \subset \mathcal{E}(B)$ and $(c-\tau, c] \times(y-\tau, y+\tau) \subset \mathcal{N}(B)$. Since $f$ is bilaterally $\mathfrak{c}$-dense in itself, the set $A=(c-\tau, c] \times\left(f(c)-\frac{\tau}{2}, f(c)+\frac{\tau}{2}\right) \cap f$ has cardinality $\mathfrak{c}$.

For every $\langle a, f(a)\rangle \in A,\left|f(a)-\left(y-\frac{\tau}{2}, y+\frac{\tau}{2}\right)\right|<\varepsilon$, so since $\langle a, f(a)\rangle \in$ $\mathcal{E}(B)$ and $\{a\} \times\left(y-\frac{\tau}{2}, y+\frac{\tau}{2}\right) \subset \mathcal{N}(B),\left|f(a)-B_{a}\right|<\varepsilon$ (see Remark 1).

Proof of Theorem 8. (1) Suppose $f \in \mathrm{AC}$ and there exists blocking set $B \subset \mathbb{I} \times \mathbb{R}$ and $\varepsilon>0$ such that $f \notin \alpha(B, \varepsilon)$.

First note, that there exist $a, b \in \mathbb{I}$ such that $a<b,\langle a, f(a)\rangle \in \mathcal{E}(B)$ and $\langle b, f(b)\rangle \in \mathcal{N}(B)$. To choose $a \in[0,1)$ with $\langle a, f(a)\rangle \in \mathcal{E}(B)$ assume $\langle 0, f(0)\rangle \notin$ $\mathcal{E}(B)$. Since $f \notin \alpha(B, \varepsilon)$ and $\{0\} \times \mathbb{R} \subset B \cup \mathcal{E}(B)$ (see Theorem 1 (1)), there exists $y_{0} \in \mathbb{R}$ such that $\left\langle 0, y_{0}\right\rangle \in \mathcal{E}(B)$ and $\left|f(0)-y_{0}\right|<\varepsilon$. Take $\tau>0$ such that $[0, \tau) \times\left(y_{0}-\tau, y_{0}+\tau\right) \subset \mathcal{E}(B)$ (by Th. $1(3), \mathcal{E}(B)$ is right-open). Since $f$ is bilaterally $\mathfrak{c}$-dense in itself and cardinality of $f \cap B$ is less than $\mathfrak{c}$, the set $A=[0, \tau) \times\left(f(0)-\frac{\tau}{2}, f(0)+\frac{\tau}{2}\right) \cap f \cap(\mathcal{E}(B) \cup \mathcal{N}(B))$ is not empty. Take $\langle a, f(a)\rangle \in A$. We will show that $\langle a, f(a)\rangle \in \mathcal{E}(B)$. Indeed, if $\langle a, f(a)\rangle \in \mathcal{N}(B)$ then, since $\left|f(a)-\left(y_{0}-\frac{\tau}{2}, y_{0}+\frac{\tau}{2}\right)\right|<\varepsilon$ and $\{a\} \times\left(y_{0}-\frac{\tau}{2}, y_{0}+\frac{\tau}{2}\right) \subset \mathcal{E}(B)$, lemma 1 implies $f \in \alpha(B, \varepsilon)$. So $\langle a, f(a)\rangle \in \mathcal{E}(B)$. Analogously we can find $b \in(a, 1]$ such that $\langle b, f(b)\rangle \in \mathcal{N}(B)$.

By Theorem 2, there exists $c \in[a, b]$ such that $f$ breaks through $B$ at $\langle c, f(c)\rangle$.

Since $f \notin \alpha(B, \varepsilon)$, there exists $y_{c} \in \mathbb{R}$ such that $\left\langle c, y_{c}\right\rangle \notin B$ and $\left|f(c)-y_{c}\right|<$ $\varepsilon$. Theorem 1 (6) shows that $\left\langle c, y_{c}\right\rangle \in \mathrm{A}_{\mathrm{EE}}(B) \cup \mathrm{A}_{\mathrm{NN}}(B) \cup \mathrm{A}_{\mathrm{NE}}(B)$. All cases are analogous, so assume $\left\langle c, y_{c}\right\rangle \in \mathrm{A}_{\mathrm{NE}}(B)$. Fix $\tau>0$ such that $(c-\tau, c] \times$ $\left(y_{c}-\tau, y_{c}+\tau\right) \subset \mathcal{N}(B)$ and $(c, c+\tau) \times\left(y_{c}-\tau, y_{c}+\tau\right) \subset \mathcal{E}(B)$. Since $f$ breaks through $B$ at $\langle c, f(c)\rangle$, there are $x_{1}<x_{2}$ with $\left\langle x_{1}, f\left(x_{1}\right)\right\rangle,\left\langle x_{2}, f\left(x_{2}\right)\right\rangle \in$ $(c-\tau, c+\tau) \times\left(f(c)-\frac{\tau}{2}, f(c)+\frac{\tau}{2}\right)$ and $\left\langle x_{1}, f\left(x_{1}\right)\right\rangle \in \mathcal{E}(B),\left\langle x_{2}, f\left(x_{2}\right)\right\rangle \in \mathcal{N}(B)$. Now, we have $x_{1} \leq c$ or $x_{2}>c$.

In the first case $\left\langle x_{1}, f\left(x_{1}\right)\right\rangle \in \mathcal{E}(B),\left\{x_{1}\right\} \times\left(y_{c}-\frac{\tau}{2}, y_{c}+\frac{\tau}{2}\right) \subset \mathcal{N}(B)$ and $\left|f\left(x_{1}\right)-\left(y_{c}-\frac{\tau}{2}, y_{c}+\frac{\tau}{2}\right)\right|<\varepsilon$, so lemma 1 implies $f \in \alpha(B, \varepsilon)$.

In the second case $\left\langle x_{2}, f\left(x_{2}\right)\right\rangle \in \mathcal{N}(B),\left\{x_{2}\right\} \times\left(y_{c}-\frac{\tau}{2}, y_{c}+\frac{\tau}{2}\right) \subset \mathcal{E}(B)$ and $\left|f\left(x_{2}\right)-\left(y_{c}-\frac{\tau}{2}, y_{c}+\frac{\tau}{2}\right)\right|<\varepsilon$, and again lemma 1 gives $f \in \alpha(B, \varepsilon)$. A contradiction.

(2) Suppose $\left\{f_{n}\right\}_{n} \subset \alpha$ uniformly converges to $f$. Let $\varepsilon>0$ and $B \subset \mathbb{I} \times \mathbb{R}$ be a blocking set.

Fix $n \in \mathbb{N}$ such that $\left|f_{n}-f\right|<\frac{\varepsilon}{2}$. Since $f_{n} \in \alpha\left(B, \frac{\varepsilon}{2}\right)$, we have two cases:

- The set $C=\left\{x|| f_{n}(x)-B_{x} \mid<\frac{\varepsilon}{2}\right\}$ has cardinality $\mathfrak{c}$. Then for every $x \in C$ we have $\left|f(x)-B_{x}\right| \leq\left|f(x)-f_{n}(x)\right|+\left|f_{n}(x)-B_{x}\right|<\varepsilon$. 
- There exists $c \in \mathbb{I}$ such that $\left[f_{n}(c)-\frac{\varepsilon}{2}, f_{n}(c)+\frac{\varepsilon}{2}\right] \subset B_{c}$. Because $f(c) \in$ $\left(f_{n}(c)-\frac{\varepsilon}{2}, f_{n}(c)+\frac{\varepsilon}{2}\right)$, so $\langle c, f(c)\rangle \in B$ and $\left(f_{n}(c)-\frac{\varepsilon}{2}, f_{n}(c)+\frac{\varepsilon}{2}\right) \subset$ $B_{c} \cap(f(c)-\varepsilon, f(c)+\varepsilon)$.

In both cases $f \in \beta \cap \mathrm{AAC}$.

(3) We will only show that $\mathrm{AAC} \subset \mathcal{U}$.

Suppose $f \in \mathrm{AAC}$ and $f \notin \mathcal{U}$. Since every function in the class $\mathcal{U}$ is $\mathfrak{c}$-dense in itself ([3]), then there exist an interval $J=[a, b]$, a set $A$ of cardinality less than $\mathfrak{c}, y \in[f(a), f(b)]$ (without lost of generality we can assume that $f(a)<f(b))$ and $\varepsilon>0$ such that $a, b \notin A,(y-3 \varepsilon, y+3 \varepsilon) \subset(f(a), f(b))$ and $f(J \backslash A) \cap(y-3 \varepsilon, y+3 \varepsilon)=\emptyset$. Since cardinality of $A$ is less than $\mathfrak{c}$, we can also find $y^{\prime} \in(y-\varepsilon, y+\varepsilon)$ such that $y^{\prime} \notin f(A)$. Now, for every $\langle x, f(x)\rangle$ define open set $S_{\langle x, f(x)\rangle}$ :

$$
\begin{aligned}
S_{\langle a, f(a)\rangle}= & {\left[0, \frac{a+b}{2}\right) \times(f(a)-2 \varepsilon, f(a)+2 \varepsilon) } \\
S_{\langle b, f(b)\rangle}= & \left(\frac{a+b}{2}, 1\right] \times(f(b)-2 \varepsilon, f(b)+2 \varepsilon) \\
S_{\langle x, f(x)\rangle}= & {[0, a) \times(f(x)-2 \varepsilon, f(x)+2 \varepsilon \text { for } x \in[0, a)} \\
S_{\langle x, f(x)\rangle}= & (b, 1] \times(f(x)-2 \varepsilon, f(x)+2 \varepsilon) \text { for } x \in(b, 1] \\
S_{\langle x, f(x)\rangle}= & (a, b) \times(f(x)-2 \varepsilon, f(x)+2 \varepsilon) \text { for } x \in(a, b) \backslash A \\
S_{\langle x, f(x)\rangle}= & (a, b) \times U_{x}, \text { where } U_{x} \text { is an open interval containing } f(x) \text { and } \\
& \quad \text { not containing } y^{\prime} \text { for } x \in(a, b) \cap A .
\end{aligned}
$$

Since $V=\bigcup_{x \in \mathbb{I}} S_{\langle x, f(x)\rangle}$ is an open neighbourhood of $f$ fullfilling all assumptions of Remark 2, there exists continuous function $g: \mathbb{I} \rightarrow \mathbb{R}, g \subset V$. We have built $V$ such that $g(a)<y^{\prime}, g(b)>y^{\prime}$ and $\left((a, b) \times\left\{y^{\prime}\right\}\right) \cap V=\emptyset$. So, $g$ cannot take the value $y^{\prime}$ between $a$ and $b$; this contradicts the fact that $g$ has the Darboux property.

Corollary 2. $\overline{\mathrm{AC}} \subset \beta \cap \mathrm{AAC}^{4}$

Corollary 3. $\overline{\mathrm{AC}}=\bar{\alpha}$.

Proof. This is a consequence of inclusions $\alpha \subset \overline{\mathrm{AC}}$ and $\mathrm{AC} \subset \alpha$.

Finally, we would like to state a problem.

Problem 1. Does the equality $\mathrm{AAC}=\mathcal{U} \cap \mathrm{AAC}_{0}$ hold?

\footnotetext{
${ }^{4}$ Using technique from the proof of Theorem 2 it is possible to show that $\mathrm{AAC} \subset \beta$, but such proof is much longer than the one presented above.
} 


\section{References}

[1] J. B. Brown, Almost continuous Darboux functions and Reed's pointwise convergence criteria, Fund. Math. LXXXVI (1974), 1-17.

[2] J. B. Brown, Almost continuity of the Cesáro-Vietoris function, Proc. Amer. Math. Soc., 49 (1975), 185-188. Fund. Math. LXXXVI (1974), 1-17.

[3] A. M. Bruckner, J. G. Ceder, M. Weiss, Uniform limits of Darboux functions, Colloq. Math., 15 (1966), 65-77.

[4] R. Gibson, T. Natkaniec, Darboux like functions, Real Anal. Exchange, 22(2) (1996-97), 492-533.

[5] R. Gibson, T. Natkaniec, Darboux like functions. Old problems and new results, Real Anal. Exchange, 24(2) (1998-99), 487-496.

[6] J. Jastrzębski, An answer to a question of R. G. Gibson and F. Roush, Real Anal. Exchange, 15 (1989-90), 340-341.

[7] K. R. Kellum, Sums and limits of almost continuous functions, Colloq. Math., 31 (1974), 125-128.

[8] K. R. Kellum, B. D. Garrett, Almost continuous real functions, Proc. Amer. Math. Soc., 33 (1972), 181-184.

[9] A. Maliszewski, Maximums of almost continuous functions, preprint.

[10] M. H. Miller, Jr., Equivalence of certain discontinuous functions under closure, Proc. Amer. Math. Soc., 54 (1976), 384-388.

[11] T. Natkaniec, Almost continuity, Real Anal. Exchange 17 (1991-92), $462-520$.

[12] J. H. Roberts, Zero-dimensional sets blocking connectivity functions, Fund. Math., LVII (1965), 173-179.

[13] H. Rosen, Closure of Darboux graphs, Real Anal. Exchange, 18 (199293), 87-94.

[14] J. Stallings, Fixed point theorems for connectivity maps, Fund. Math., 47 (1959), 249-263. 